\title{
Standard chemoradiation versus intensity-modulated chemoradiation: a quality of life assessment in oropharyngeal cancer patients
}

\author{
Sarah E. Mowry $\cdot$ Christopher Tang $\cdot$ Ahmad Sadeghi $\cdot$ \\ Marilene B. Wang
}

Received: 28 September 2009 / Accepted: 15 December 2009 / Published online: 29 December 2009

(C) The Author(s) 2009. This article is published with open access at Springerlink.com

\begin{abstract}
This study is based on the context that many patients with advanced oropharyngeal carcinoma are being treated with primary chemoradiation. The aims of this study are to identify differences in quality of life (QOL) between patients with advanced oropharyngeal cancer following traditional chemoradiation versus chemotherapy with intensity-modulated radiation therapy (CIMRT). This research is designed on a cohort study from an academic tertiary referral center. Fifty patients were identified from an institutional database of patients who had undergone primary chemotherapy and radiation (traditional or IMRT) for advanced oropharyngeal carcinoma. Patients responded via mail using the University of Washington quality of life instrument version 4. Statistical analysis of data was performed using Chi-square and Wilcoxon tests. The results comprise the responses of 17 CRT (57\%) and 14 CIMRT (70\%) patients. The patients completed the survey between
\end{abstract}

This paper was read at the American Academy of OtolaryngologyHead and Neck Surgery Annual meeting in Chicago, IL, USA, on September 23, 2008.

S. E. Mowry $(\varangle) \cdot$ C. Tang $\cdot$ M. B. Wang

Division of Head and Neck Surgery,

David Geffen School of Medicine, University of California,

Los Angeles, 62-132 CHS, BOX 951624,

Los Angeles, CA 90095-1624, USA

e-mail: Smowry1978@gmail.com

\section{A. Sadeghi}

Department of Radiation Oncology,

VA Greater Los Angeles Healthcare System,

Los Angeles, USA

\section{B. Wang}

Department of Surgery,

VA Greater Los Angeles Healthcare System,

Los Angeles, USA
9 and 44 months following end of treatment. When adjusted for tumor stage and time since treatment, CIMRT patients reported improved appearance $(p=0.05)$, chewing $(p=0.02)$, and $\operatorname{mood}(p=0.01)$. There was a trend toward significance for improved activity $(p=0.07)$, recreation $(p=0.07)$, and anxiety $(p=0.08)$. There were no differences between the two groups for saliva, taste, shoulder function, speech, and swallowing. But there was a trend for significance for improved overall QOL in patients who had undergone CIMRT $(p=0.06)$. In conclusion, CIMRT results in improved QOL for some domains but surprisingly not for swallowing or saliva. Patients undergoing CIMRT also report slightly better QOL overall when compared to patients receiving more traditional forms of radiation therapy.

Keywords IMRT $\cdot$ Chemoradiation · Oropharyngeal cancer · Quality of life

\section{Introduction}

The landmark Veterans Administration Laryngeal Cooperative study published in 1991 initiated the era of organ preservation [1]. Today, many patients are advised to choose chemoradiation as their primary treatment for advanced oropharyngeal carcinomas (stages III-IV) [2, 3].

Traditional radiotherapy involves opposed port beams directed at the tumor. This type of radiation affects all the surrounding tissues as well. In oropharyngeal lesions, this typically results in high doses to the parotids, submandibular glands, and the mandible (>60 Gy). The resulting longterm complications of xerostomia and osteoradionecrosis are well known and negatively impact patients' quality of life (QOL). Recent advances in computational power have 
allowed radiation therapy to be directed more specifically to the tumor bed. This conformational therapy is referred to as intensity-modulated radiotherapy (IMRT). The goal of using IMRT is to limit the radiation dose to radiosensitive structures, such as the parotids and the mandible. Within the literature, studies have demonstrated improved dosimetry to salivary structures with resultant-improved salivary flow [4]. The literature has demonstrated that IMRT protocols provide equivalent control rates and overall survival when compared to more traditional opposed port therapy [5]. However, it is unclear how the improved dosimetry impacts QOL of long-term head and neck cancer survivors.

The other component of the organ preservation protocols for oropharyngeal carcinoma is chemotherapy. The protocols for this treatment are still being perfected, but the mainstay of chemotherapy for head and neck carcinomas involves 5-flurouracil, a nucleotide analog, and cisplatin-a DNA chelating agent. Docetaxel is also now being added to this regimen [6]. The side effects of these agents can be severe and include neutropenia, mucositis, renal toxicity, neuropathy, and hearing loss. Terrell et al. [7] demonstrated that the addition of chemotherapy to treatment negatively impacts QOL of patients.

QOL is a subjective perception by the patient that includes physical, emotional, and social well being. It is well established that different subsites within the head and neck have different perceived QOL following treatment; patients with oropharyngeal carcinoma tend to report worse QOL than do other head and neck cancer patients and have poorer functional outcomes $[8,9]$. Furthermore, specific aspects of treatment greatly affect a patient's QOL. The need for gastrostomy and tracheostomy tubes, comorbid medical conditions, and need for a neck dissection all negatively affect QOL outcomes [7].

A previous study demonstrated no difference in QOL outcomes between patients who had undergone chemoradiation versus surgery and radiation for advanced oropharyngeal carcinoma [10]. In the current study a comparison between QOL outcomes between patients who received chemotherapy and IMRT (CIMRT) versus standard chemoradiation with opposed port therapy (CRT) was undertaken. The hypothesis of this study is that CIMRT patients experience improved QOL compared to those undergoing CRT.

\section{Materials and methods}

An institutional review board protocol was approved for this study. Patients were recruited from a tertiary referral center from a registry of patients who had undergone radiation therapy of all types from 2001 to 2006. Patients had biopsy-proven squamous cell carcinoma of the oropharynx (including tonsil, base of tongue, or pharynx). These tumors were clinically staged as stage II through IV based on criteria described by the American Joint Committee on Cancer (AJCC). All patients involved in the study had undergone either primary concurrent chemoradiation (CRT) or concurrent chemotherapy with IMRT (Table 1). Patients were excluded if they required surgical salvage after CRT/CIMRT or had metastatic disease at the time of diagnosis.

A total of 50 patients identified from institutional databases met eligibility requirements for the study. All eligible patients were mailed copies of the University of Washington Quality of Life Questionnaire, version four (UW-QOLv4). Patients were also contacted by telephone to encourage participation. Patients voluntarily filled out the questionnaire at home and mailed their responses to the

Table 1 Chemotherapy and radiation protocol for study participants

\begin{tabular}{|c|c|c|c|c|}
\hline ID no. & TNM & XRT type & $\begin{array}{l}\text { Total XRT } \\
\text { dose }\end{array}$ & Chemo \\
\hline 1 & $\mathrm{~T} 4 \mathrm{~N} 1$ & Conventional & 7,020 & Cisplatin/etoposide \\
\hline 2 & $\mathrm{~T} 3 \mathrm{~N} 2 \mathrm{~b}$ & Conventional & 7,020 & $2 \times$ cisplatin $/ 5 \mathrm{FU}$ \\
\hline 3 & T3N2c & Conventional & 7,000 & $2 \times$ cisplatin $/ 5 \mathrm{FU}$ \\
\hline 4 & T1N1 & Conventional & 7,200 & $1 \times$ cisplatin $/ 5 \mathrm{FU}$ \\
\hline 5 & T2N0 & Conventional & 7,000 & $2 \times$ carbo $/ 5 \mathrm{FU}$ \\
\hline 6 & $\mathrm{~T} 3 \mathrm{~N} 2$ & Conventional & 7,020 & $2 \times$ cisplatin $/ 5 \mathrm{FU}$ \\
\hline 7 & $\mathrm{~T} 3 \mathrm{~N} 2 \mathrm{~b}$ & Conventional & $5,400^{\mathrm{a}}$ & $2 \times$ cisplatin $/ 5 \mathrm{FU}$ \\
\hline 8 & $\mathrm{~T} 1 \mathrm{~N} 2 \mathrm{~b}$ & Conventional & 7,200 & $2 \times$ cisplatin $/ 5 \mathrm{FU}$ \\
\hline 9 & T4N1 & Conventional & 7,380 & $2 \times$ cisplatin $/ 5 \mathrm{FU}$ \\
\hline 10 & $\mathrm{~T} 2 \mathrm{~N} 1$ & Conventional & 7,000 & $2 \times$ cisplatin $/ 5 \mathrm{FU}$ \\
\hline 11 & T4N1 & Conventional & 7,020 & $2 \times$ cisplatin $/ 5 \mathrm{FU}$ \\
\hline 12 & T4N3 & Conventional & 7,020 & $2 \times$ cisplatin $/ 5 \mathrm{FU}$ \\
\hline 13 & T4N1 & Conventional & 7,560 & $3 \times$ cisplatin \\
\hline 14 & $\mathrm{~T} 3 \mathrm{~N} 3$ & Conventional & NA & NA \\
\hline 15 & T4N0 & Conventional & NA & NA \\
\hline 16 & T4N0 & Conventional & 7,020 & $2 \times$ cisplatin $/ 5 \mathrm{FU}$ \\
\hline 17 & $\mathrm{~T} 4 \mathrm{~N} 3$ & IMRT & 7,000 & $2 \times$ cisplatin \\
\hline 18 & $\mathrm{~T} 2 \mathrm{~N} 0$ & IMRT & 7,000 & $2 \times$ cisplatin \\
\hline 19 & $\mathrm{~T} 2 \mathrm{~N} 2 \mathrm{a}$ & IMRT & 7,000 & $3 \times$ cisplatin $/ 5 \mathrm{FU}$ \\
\hline 20 & $\mathrm{~T} 1 \mathrm{~N} 2 \mathrm{~b}$ & IMRT & 7,000 & $2 \times$ cisplatin $/ 5 \mathrm{FU}$ \\
\hline 21 & $\mathrm{~T} 2 \mathrm{~N} 1$ & IMRT & 7,000 & $2 \times$ cisplatin \\
\hline 22 & $\mathrm{~T} 2 \mathrm{~N} 2$ & IMRT & 6,800 & $3 \times$ cisplatin \\
\hline 23 & $\mathrm{~T} 2 \mathrm{~N} 0$ & IMRT & 7,000 & $2 \times$ cisplatin \\
\hline 24 & $\mathrm{~T} 2 \mathrm{~N} 2 \mathrm{~b}$ & IMRT & 7,000 & $4 \times$ cisplatin \\
\hline 25 & $\mathrm{~T} 4 \mathrm{~N} 3$ & IMRT & 7,000 & $2 \times$ cisplatin \\
\hline 26 & $\mathrm{~T} 3 \mathrm{~N} 2$ & IMRT & 6,800 & ERBITUX \\
\hline 27 & $\mathrm{~T} 1 \mathrm{~N} 2 \mathrm{~b}$ & IMRT & 7,000 & $2 \times$ cisplatin \\
\hline 28 & $\mathrm{~T} 3 \mathrm{~N} 2 \mathrm{~b}$ & IMRT & 7,000 & $2 \times$ cisplatin \\
\hline 29 & $\mathrm{~T} 2 \mathrm{~N} 0$ & IMRT & 6,800 & None \\
\hline 30 & $\mathrm{~T} 3 \mathrm{~N} 1$ & IMRT & 6,800 & $2 \times$ cisplatin \\
\hline
\end{tabular}

NA records not available, $5 F U 5$ fluorouracil, carbo carboplatin

a Patient moved from area before finishing treatment 
study organizers. Questionnaires were identified by a nondescript number, and data were entered into a confidential database.

The UW-QOLv4 instrument is scored on a scale of $0-100$ for the first and third segments. The first section consists of 12 domains pertaining to the degree of QOL in the categories of pain, appearance, activity, recreation, swallowing, chewing, speech, shoulder function, taste, saliva, mood, and anxiety. A score of 0 indicates very poor or no functional capacity with regard to that domain while a score of 100 indicates no disability in that domain. The second segment of the questionnaire asks patients to choose three of the above domains that have been the most important to them in the past 7 days. In the final part of the instrument, patients are given three general questions comparing their (1) current health-related quality of life (HR-QOL) to 1 month before developing cancer, (2) HR-QOL during the past 7 days, and (3) overall QOL during the past 7 days. The final segment is scored with 0 indicating very poor QOL and 100 indicating outstanding QOL, with a range of scores between.

Statistical analysis of the responses was conducted using the Wilcoxon rank sum test and the Chi-square test. Results were adjusted to control for time from treatment to questionnaire and for tumor stage.

\section{Results}

Twenty-nine questionnaires were returned (58\% return rate), with $16 / 30$ patients in the CRT cohort and $13 / 20$ in the CIMRT cohort. All patients were male. Eleven of 16 patients in the CRT cohort and 9 of 14 patients in the CIMRT group had stage IV tumors. Each group had one patient who was staged with T2N0 cancer. There was no statistically significant difference in the tumor stages between the two groups. All patients received chemotherapy concomitantly with their radiation. No patient received induction chemotherapy. One patient in the CIMRT cohort received cetuximab rather than 5-fluorouracil or cisplatin. Table 1 shows the various radiation doses and chemotherapy regimens for the responding patients. The average time between the completion of treatment and questionnaire for the CIMRT group was 13 months (range 4-22 months) while that for the CRT group was 25 months (range 4-53 months).

Patients who received CIMRT reported statistically significantly better QOL with regard to appearance $(p=0.05)$, chewing $(p=0.02)$, and mood $(p=0.01)$. There was a trend toward significance in favor of CIMRT with regard to anxiety $(p=0.08)$, recreation $(p=0.07)$, and activity $(p=0.07)$. No statistically significant difference was reported between the two cohorts with respect to saliva $(p=0.34)$, pain ( $p=0.47)$, swallowing $(p=0.29)$, speech $(p=0.50)$, shoul$\operatorname{der}(p=0.24)$, and taste $(p=0.32)$.
With regard to overall QOL and HR-QOL, there was no difference between the two groups $(p=0.31$ and 0.09 , respectively). However, when asked about QOL compared to 1 month before the development of cancer, CIMRT patients reported improved QOL $(p=0.06)$.

\section{Discussion}

Primary chemotherapy with radiation is a popular treatment option for patients with advanced oropharyngeal carcinoma. Recent advances have allowed for conformal radiation treatment strategies in order to minimize complications in radiosensitive structures. QOL in long-term survivors is an important outcome measure and is currently an active area of research. In this study, QOL outcomes were compared in a cohort study between traditional opposed port radiotherapy and IMRT; both groups received concomitant chemotherapy. Overall QOL was not different in the CIMRT patients; however, several domains did demonstrate improved QOL as measured by the UW-QOLv4. Appearance, chewing, and mood were better for CIMRT patients. No difference was detected for all other domains, including saliva, taste, and swallowing.

Specific evaluation of the global and HR-QOL in patients treated for oropharyngeal carcinoma with IMRT is limited. The majority of studies available evaluate xerostomia and xerostomia-related QOL [11, 12]. Of the studies that evaluate global QOL, it has been demonstrated that IMRT patients experience improved QOL [13, 14].

Yao et al. [13] recently published the only study focused on oropharyngeal cancer survivors specifically. They evaluated 56 patients with the Head and Neck Cancer Inventory which measures HR-QOL and questions about their diet. They found that eating and dysphagia scores were significantly improved in the IMRT group of patients. Other domains demonstrated improved QOL scores for IMRT patients but did not reach statistical significance. Furthermore, 1 year after treatment the IMRT patients' scores continued to improve while the CRT patients' scores had reached a plateau [13]. In the current study, a different head and neck specific instrument was used, specifically the UW-QOLv4; thus it is somewhat difficult to compare the data. However, it is apparent there were differences between the findings of the Iowa group and our cohort. In the present study, only 3 of 12 domains demonstrated significance although several domains approached significance. Specifically, patients did not report improved saliva or swallowing in our study while Yao et al. [13] did report improvement for these domains.

Other studies in the literature have looked primarily at either xerostomia-related QOL or dysphagia-related QOL. Levendag et al. [15] studied the dosimetry to the constrictor 
muscles in oropharyngeal carcinoma patients and determined that higher doses delivered to these muscles resulted in higher rates of severe dysphagia and gastrostomy dependence. Their study is somewhat difficult to interpret because not all patients received chemotherapy, which may lead to increased scarring in the pharyngeal musculature. In our study, patients received relatively uniform chemotherapy regimens and similar dosimetry to tumor. The patients in our cohorts did not report improved swallowing or saliva, but CIMRT patients did report better chewing. The noted improvement in chewing may be explained by the sparing of the intrinsic musculature of the tongue and other oral structures with IMRT. The ability to manipulate the bolus within the oral cavity is an integral part of the oral phase of chewing. Improved tongue mobility due to less radiation induced fibrosis potentially accounts for this result.

The results for saliva are somewhat surprising based on the radiation protocol in effect at our institution. All patients with oropharyngeal primary tumors who undergo IMRT receive $70 \mathrm{~Gy}$ to the gross tumor. Sites within the oropharynx or neck with likely microscopic disease receive $63 \mathrm{~Gy}$ and sites with potential or remote disease receive $56 \mathrm{~Gy}$. The parotid glands are carefully identified and mapped so that $50 \%$ of each gland received only $20 \mathrm{~Gy}$. The rest of the gland receives doses from 56 to $70 \mathrm{~Gy}$ (see Fig. 1). With approximately half of the gland spared from high dose radiation, recovery of salivary function is expected within several months after the completion of treatment. Conversely, patients treated with conventional (opposed port) radiation do not have sparing of the parotid glands. For treatment of the oropharynx, the parotids receive 70 Gy to the entire gland on each side. Minimal salivary function is expected to return in these patients. Given the differences between the radiation protocols, the lack of difference regarding salivary QOL is surprising. Perhaps those undergoing IMRT have decreased perception of salivary flow when compared to pre-therapy flow rates and thus report decreased salivary QOL.

Patients receiving CIMRT did report significantly better mood and appearance than their CRT counterparts. The cause of this difference is unclear. Perhaps there is less "woody induration" in IMRT patients, which results in a more supple appearance to the neck contour and hence an improved perception of appearance. The results with regard to mood were unexpected and cannot be explained by a difference either in final XRT dose or in chemotherapy regimens, as these were similar between the groups. QOL is, by definition, a subjective evaluation of the patient's perception. These results are inherently biased and dependent on the patient's expectations of their treatment and its outcomes. Perhaps, the CIMRT patients reported better mood because they expected to feel better after treatment.

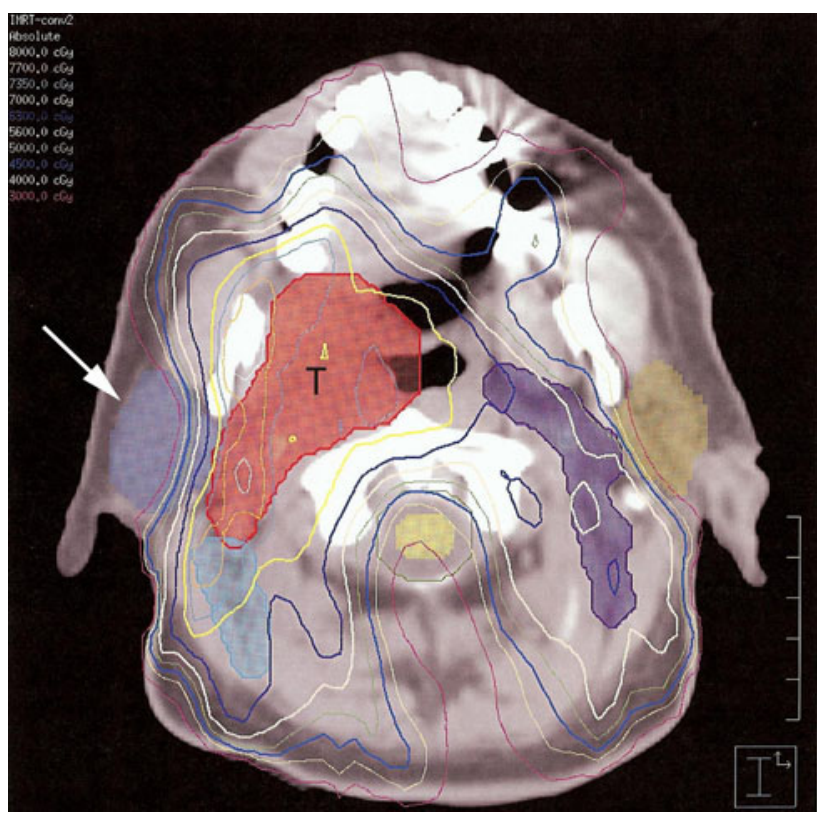

Fig. 1 Radiation planning diagram for T4 carcinoma of the right tonsil. The primary tumor volume receives $70 \mathrm{~Gy}$ (marked $T$ and shaded in $r e d$ ). The radiation plan is designed to spare $50 \%$ of each parotid gland (blue-shaded area identified by the arrowhead). The dose distribution lines show rapid falloff in the radiation dose between the tumor and the majority of the parotid gland. A small amount of the gland receives high dose radiation where the tumor approaches the gland

Other differences in the perceived QOL of these patients may be elucidated with a different QOL instrument. There are a number of head and neck specific questionnaires available which have been validated in the literature including the HR-QOL, EORTC-C30 with the EORTC-H\&N35, and UW-QOL. A recent review by Tschiesner et al. [16] compared these instruments using the international classification of function, disability, and health (ICF) schema. Using this schema, the content of the instruments can be more easily compared as the instrument content was correlated with specific ICF concepts. The EORTC questionnaires cover a broader range of ICF categories when compared to the UW-QOL. The EORTC instrument includes questions regarding memory and attention functions; sexual functions; respiratory functions; a variety of activities of daily living such as toileting and dressing; and smell. This instrument is used widely in Europe but less commonly in the United States. However, both instruments have approximately $70 \%$ of their content that can be correlated to an ICF category; the EORTC instrument covers 43 categories while the UW-QOL covers 23 categories [16]. Two of the major benefits of the UW-QOL, and the reason it was chosen for this study, are its broad range of questions and the relative ease of completion. In order to encourage participation in the veteran population from which this cohort was drawn, an instrument which could be completed 
quickly but covered a broad range of topics was required. The UW-QOL can be completed in a quarter of the time needed to complete the EORTC instruments.

The differences between the two radiation groups may be helpful when deciding which radiation modality to use for specific patients. Those patients with comorbid conditions report having significantly worse QOL prior to beginning therapy than those without these conditions [17]. In these patients, all other factors being equal, CIMRT may be a better option to try to minimize the negative impact of therapy on their already diminished QOL.

Other treatment options for advanced oropharyngeal carcinoma include surgery with adjuvant radiation (or chemoradiation). QOL outcomes between surgery and opposed port chemoradiation have been previously described [10]. Surgical therapy is associated with late toxicity, including scarring, fibrosis, laryngeal, and esophageal stenoses, all of which also affect QOL for head and neck cancer patients. As both appearance and chewing can be significantly impacted by surgery, it is possible that patients who undergo primary CIMRT may have improved QOL compared with patients who undergo surgery and radiation. Although there were no differences noted between the two chemoradiation groups in the aforementioned study, the impact of CIMRT on QOL when compared to surgery and radiation is yet to be explored. Another treatment option that is available is induction chemotherapy followed by radiation. While none of the patients in this study received induction chemotherapy, it is known that chemotherapy, in general, negatively impacts on QOL [7]. How the timing of chemotherapy, induction, or concomitant, impacts QOL has not been explored. It seems likely that although early toxicity of therapy may be diminished in those patients undergoing induction chemotherapy, the long-term effects on QOL would be similar to those receiving concomitant chemoradiation.

This current study is limited in several ways. One limitation to this study was that our sample size was small, and there may not have been sufficient power to find significant differences between the two groups. A larger number of patients may demonstrate subtle differences between the groups and may result in statistically significant differences for anxiety, recreation, and activity. An ideal study would be a prospective longitudinal one involving a larger number of patients, and in future such studies are planned. However, we believe that this current study is a valuable pilot study which yields important information about what oropharyngeal cancer patients should expect from CRT and CIMRT, especially when discussing the benefits of a given treatment. It is important not to overstate the benefits of that treatment and ensure that patients have realistic expectations for their post treatment QOL.

\section{Conclusion}

Patients who receive chemotherapy and IMRT do have improvement in certain domains of QOL, specifically appearance, mood, and chewing. However, with regard to overall QOL, CIMRT and CRT patients have similar perceptions of QOL. Surprisingly, in our study CIMRT patients did not report better saliva or swallowing when compared to CRT patients. Although studies in the literature demonstrate improved salivary flow rates with parotid sparing radiation regimens, this does not appear to have improved the patients' perceptions of dry mouth following treatment. This information is important to understand when counseling patients regarding the expected treatment outcomes.

Open Access This article is distributed under the terms of the Creative Commons Attribution Noncommercial License which permits any noncommercial use, distribution, and reproduction in any medium, provided the original author(s) and source are credited.

\section{References}

1. Induction chemotherapy plus radiation compared with surgery plus radiation in patients with advanced laryngeal cancer. The Department of Veterans Affairs Laryngeal Cancer Study Group (1991) N Eng J Med 324(24):1685-1690

2. Calais G, Alfonsi M, Bardet E, Sire C, Germain T, Bergerot P et al (1999) Randomized trial of radiation therapy versus concomitant chemotherapy and radiation therapy for advanced-staged oropharyngeal carcinoma. J Natl Cancer Inst 91(24):2081-2086

3. Machtay M, Rosenthal DI, Hershock D, Jones H, Williamson S, Greenberg MJ et al (2002) Organ preservation therapy using induction plus concurrent chemoradiation for advanced resectable oropharyngeal carcinoma: a University of Pennsylvania phase II trial. J Clin Oncol 20(19):3964-3971

4. Münter MW, Hoffner S, Hof H, Herfarth KK, Haberkorn U, Rudat $\mathrm{V}$ et al (2007) Changes in salivary gland function after radiotherapy of head and neck tumors measured by quantitative pertechnetate scintigraphy: comparison of intensity-modulated radiotherapy and conventional radiation therapy with and without Amifostine. Int J Radiat Oncol Biol Phys 67(3):651-659

5. Lee NY, de Arrudo FF, Puri DR, Wolden SL, Narayana A, Mechaloakos J (2006) A comparison of intensity-modulated radiation therapy and concomitant boost radiotherapy in the setting of concurrent chemotherapy for locally advanced oropharyngeal carcinoma. Int J Radiat Oncol Biol Phys 66(4):966-974

6. Calais G, Bardet E, Sire C, Alfonsi M, Bourhis J, Rhein B et al (2004) Radiotherapy with concomitant weekly docetaxel for Stages III/IV oropharynx carcinoma. Results of the 98-02 GORTEC Phase II trial. Int J Radiat Oncol Biol Phys 58(1):161-166

7. Terrell JE, Ronis DL, Fowler KE, Bradford CR, Chepeha DB, Prince ME et al (2004) Clinical predictors of quality of life in patients with head and neck cancer. Arch Otolaryngol Head Neck Surg 130(4):401-408

8. Pourel N, Peiffert D, Lartigau E, Desandes E, Luporsi E, Conroy T (2002) Quality of life in long-term survivors of oropharynx carcinoma. Int J Radiation Oncology Biol Phys 54(3):742-751

9. Ferlito A, Rogers SN, Shaha AR, Bradley PJ, Rinaldo A (2003) Quality of life in head and neck cancer. Acta Otolaryngol 123(1):5-7 
10. Mowry SE, Ho A, Lotempio MM, Sadeghi A, Blackwell KE, Wang MB (2006) Quality of life in advanced oropharyngeal carcinoma after chemoradiation versus surgery and radiation. Laryngoscope 116(9):1589-1593

11. Braam PM, Terhaard CH, Roesink JM, Raaijmakers CP (2006) Intensity-modulated radiotherapy significantly reduces xerostomia compared with conventional radiotherapy. Int J Radiat Oncol Biol Phys 66(4):975-980

12. Lin A, Kim HM, Terrell JE, Dawson LA, Ship JA, Eisbruch A (2003) Quality of life after parotid-sparing IMRT for head-andneck cancer: a prospective longitudinal study. Int J Radiat Oncol Biol Phys 57(1):61-70

13. Yao M, Karnell LH, Funk GF, Lu H, Dornfeld K, Buatti JM (2007) Health-related quality-of-life outcomes following IMRT versus conventional radiotherapy for oropharyngeal squamous cell carcinoma. Int J Radiat Oncol Biol Phys 69(5):1354-1360

14. Jabbari S, Kim HM, Feng M, Lin A, Tsien C, Elshaikh M et al (2005) Matched case-control study of quality of life and xerostomia after intensity-modulated radiotherapy or standard radiotherapy for head-and-neck cancer: initial report. Int J Radiat Oncol Biol Phys 63(3):725-731

15. Levendag PC, Teguh DN, Voet P, van der Est H, Noever I, de Kruijf WJ et al (2007) Dysphagia disorders in patients with cancer of the oropharynx are significantly affected by the radiation therapy dose to the superior and middle constrictor muscle: a dose-effect relationship. Radiother Oncol 85(1):64-73

16. Tschiesner U, Rogers SN, Harreus U, Berghaus A, Cieza A (2008) Content comparison of quality of life questionnaires used in head and neck cancer based on the international classification of functioning, disability and health: a systematic review. Eur Arch Otorhinolaryngol 265(6):627-637

17. Borggreven PA, Verdonck-de Leeuw IM, Muller MJ, Heiligers ML, de Bree R, Aaronson NK, Leemans CR (2008) Quality of life and functional status in patients with cancer of the oral cavity and oropharynx: pretreatment values of a prospective study. Eur Arch Otorhinolaryngol 264(6):651-657 